\title{
Reduction of serum free fatty acids and triglycerides by liver-targeted expression of long chain acyl-CoA synthetase 3
}

\author{
MINHAO WU ${ }^{1,2}$, AIQIN CAO ${ }^{1,2}$, BIN DONG $^{1,2}$ and JINGWEN LIU ${ }^{1}$ \\ ${ }^{1}$ Veterans Affairs Palo Alto Health Care System, Palo Alto, CA 94304; ${ }^{2}$ Division of Endocrinology, \\ Department of Medicine, Stanford University, Stanford, CA 94305, USA
}

Received November 22, 2010; Accepted December 29, 2010

DOI: $10.3892 / \mathrm{ijmm} .2011 .624$

\begin{abstract}
ACSL3 is a member of the long chain acyl-CoA synthetase (ACSL) family that consists of 5 isozymes responsible for cellular fatty acid metabolism in various tissues in an isozyme-specific manner. Our previous studies have demonstrated that expression of ACSL3 mRNA and protein in liver was specifically increased after feeding hamsters with a fat- and cholesterol-enriched diet, providing the first in vivo evidence for the regulated expression of ACSL3 in liver tissue. The aim of the current study was to further investigate the role of ACSL3 in regulating hepatic lipid metabolism in vitro and in vivo. We utilized an adenoviral-mediated gene delivery approach to exogenously express hamster ACSL3 in hamster liver as well as in HepG2 cells. Transduction of HepG2 cells with Ad-hamACSL3 adenovirus elevated total cellular ACSL enzyme activity, which was accompanied by a significant reduction of cellular contents of triglycerides and total phospholipids. Immunostaining and confocal microscopy studies revealed that ACSL3 was localized to endoplasmic reticulum and mitochondria. In vivo, infection of hamsters with Ad-hamACSL3 led to sustained expression of ACSL3 mRNA and protein in liver two weeks after infection. Importantly, compared with Ad-GFP control virus infected hamsters, we observed significantly lower free fatty acids and triglycerides plus modest reduction of phospholipids in the serum of Ad-hamACSL3 infected animals. Furthermore, triglyceride levels were significantly reduced in Ad-hamACSL3 infected hamster liver. Altogether, these results provide important and physiologically relevant evidence that strengthens the link between ACSL3 expression and hepatic reduction of triglycerides and fatty acids.
\end{abstract}

Correspondence to: Dr Jingwen Liu, Veterans Affairs Palo Alto Health Care System, 3801 Miranda Avenue, Palo Alto, CA 94304, USA

E-mail: Jingwen.Liu@va.gov

Abbreviations: ACSL3, long-chain acyl-CoA synthetase 3; TG, triglyceride; FFA, free fatty acid; OM, oncostatin M

Key words: ACSL3, adenovirus, triglyceride, free fatty acids, hamster

\section{Introduction}

Long-chain acyl-CoA synthetases (ACSLs) convert fatty acids (FAs) of C12-C20 into long-chain acyl-CoAs (1) and, therefore, play key roles in lipid metabolism. Currently, there are five known isoforms of ACSL in mammal: ACSL1, ACSL3, ACSL4, ACSL5 and ACSL6 (2-6). They share a common structure that consists of five regions: an N-terminal region, luciferase-like regions 1 and 2 (that contain amino acids highly conserved among the members of the luciferase family), a linker connecting the two luciferase-like regions, and a C-terminal region. Recent studies have shown that each member of the ACSL family exhibits unique tissue expression patterns (2-8). In addition, biochemical studies have revealed that all five ACSL isoforms differ in substrate preference (2-6).

In the past decade, considerable effort has been made to gain further insight into the functional roles of individual ACSL isoforms in cellular lipid metabolism. Data collected by using the ACSL inhibitor triascin C, a fungal-derived competitive inhibitor of ACSL1, 3 and 4, provided initial evidence, although indirect, for ACSL-mediated channeling of FAs into distinct metabolic pathways (9-12). Subsequently, direct evidence resulting from both gain- and loss-of-function studies further supported the roles of individual ACSL isoforms in FA partitioning within the cell. In the liver, a major organ for lipid and energy metabolism, the predominant ACSL isoforms are ACSL1, ACSL4 and ACSL5 (2-6). Relative to ACSL4 and 5 (4,13), more studies have been carried out to characterize the function of ACSL1 in the liver using liverderived cell lines, primary hepatocytes and rodents. In vitro, adenoviral-mediated overexpression of rat ACSL1 in HepG2 cells increased triglyceride (TG) content without significantly altering fatty acid oxidation (14). Furthermore, overexpression of ACSL1 in rat primary hepatocytes increased FA reacylation and channeled FA toward diacylglyceride and phospholipid synthesis and away from cholesterol ester synthesis (15). Consistent with in vitro findings, ACSL1 overexpression via adenovirus infection elevated TG in mouse liver and increased palmitate clearance into liver TG in rats (14). Moreover, transgenic mice with heart-specific expression of ACSL1 exhibited impaired cardiac lipid homeostasis with marked accumulation of triglyceride and phospholipids in the heart (16). Together, overexpression studies supported a role of ACSL1 in channeling FAs into TG synthesis and lipid storage 
pathways in liver. However, unlike the gain-of-function data, a recent mouse model with a liver-specific knockout of ACSL1 revealed that the content of TG did not change, neither in liver nor in serum, regardless of the diets (17). Moreover, in contrast to the liver knockout, depletion of ACSL1 in adipose tissue, reduced $\mathrm{FA}$ oxidation rates, thereby suggesting a role of ACSL1 in channeling FAs toward B-oxidation in adipocytes (18). Thus, these various findings suggest that ACSL1, and perhaps other ACSL family members, may affect FA metabolism in a tissue-specific manner. It also raised the possibility that other members of the ACSL family could be equally important as ACSL1 in hepatic lipid metabolism.

We have been interested in the ACSL3 isoform since our initial report demonstrating that the expression of ACSL3 and ACSL5 in HepG2 cells was increased by cytokine oncostatin M (OM), which was associated with reduced TG synthesis and enhanced FA ß-oxidation (19). ACSL3 cDNA was originally cloned from a rat brain cDNA library (6) and its human counterpart was subsequently located to chromosome region $2 q 34-q 35$ (20). It is highly expressed in brain and has low abundance in liver (3). To facilitate our further investigations on the involvement of ACSL3 in OM-induced hypolipidemia in hyperlipidemic hamsters, we cloned the hamster ACSL3 cDNA coding sequence and showed that hamster ACSL3 sequence is highly homologous to human, mouse and rat sequences (8). The hamster ACSL3 protein has 720 amino acids with a molecular weight of $80 \mathrm{kDa}$. When compared to rat, the hamster ACSL3 exhibits a similar tissue expression pattern, at both mRNA and protein levels, with the highest expression in brain and testis and moderate expression in liver and adipose tissue (8). When ACSL3 expression was examined in various tissues of hamsters fed a normal diet and a fat- and cholesterol-enriched diet, we unexpectedly observed that both the mRNA and the protein of ACSL3 were specifically increased in the liver by the fat and cholesterol-enriched diet (8). Moreover, our laboratory recently demonstrated that ACSL3 gene expression is transcriptionally regulated by PPARS via two imperfect PPRE motifs located in ACSL3 gene promoter, and activation of PPAR $\delta$ in hamsters leads to increased ACSL3 in liver (21). Taken together, these findings suggest that, in spite of its low abundance, ACSL3 may play an important role in FA and TG metabolism in liver.

The current study was aimed at examining the direct impact of liver-targeted overexpression of ACSL3 on liver and serum lipid metabolism in vivo. We took the advantage of the liver-homing property of adenovirus (22-24) and constructed an Ad-hamACSL3 adenovirus that carries hamster ACSL3 coding sequence. We show that Ad-hamACSL3 significantly reduced cellular TG content as well as total phospholipids in transduced HepG2 cells. In hamsters, Ad-ham ACSL3 infection lowered serum TG and free fatty acids levels as well as hepatic TG levels when compared to those of the control virus (Ad-GFP) infection. These results provide new evidence to strengthen the link between ACSL3 expression and reduction of TG synthesis in the liver.

\section{Materials and methods}

Construction of Ad-hamACSL3 recombinant adenovirus. Coding sequence for hamster ACSL3 was subcloned, at the
SalI and XhoI sites, into a pShuttle-IRES-hrGFP-1 vector that contains three contiguous copies of the Flag epitope (Stratagene, CA). The resulting shuttle vector was then recombined with a pAdEasy-1 vector (Stratagene) to generate the recombinant adenoviral plasmid DNA. Ad-hamACSL3 virus was produced by transfecting the recombined adenoviral DNA into AD-293 cells. A control virus, Ad-GFP, was produced using the same method. The crude viral stocks were further amplified and purified using an Adeno-X Maxi purification kit (Clontech, CA). Viral stocks were stored at $-80^{\circ} \mathrm{C}$.

Cell culture and viral transduction. The HepG2 human hepatoma cell line was purchased from American Type Culture Collection (ATCC, MD). Cells were grown in MEM medium supplemented with $10 \%$ FBS, $2 \mathrm{mM}$ glutamine and $1 \%$ penicillin-streptomycin mixture. For viral transduction, HepG2 cells were seeded at $2 \times 10^{6}$ cells/well in 6 -well plates and allowed to grow overnight. The next day, cells were transduced with the adenovirus at various multiplicity of infection (MOIs) in $1 \mathrm{ml}$ medium containing $0.5 \%$ FBS for $2 \mathrm{~h}$. Then viralcontaining medium was replaced by fresh complete medium and cells were cultured for indicated time before harvesting.

Cellular TG and phospholipid extraction. Cellular total lipids from HepG 2 cells were extracted by $1 \mathrm{ml}$ hexane:isopropanol (3:2) twice. The extraction was evaporated under nitrogen and dissolved in isopropanol containing $10 \%$ Triton-X100 for TG and phospholipids measurement. After lipid extraction, the cells were lysed in $1 \mathrm{ml} 0.1 \mathrm{~N} \mathrm{NaOH}$ for protein concentration determination.

ACSL activity assay. Cell and tissue homogenate preparation and ACSL enzymatic activity measurement were performed as previously described (19).

Adenoviral infection in hamster. Male Golden Syrian hamsters (6-8-week-old) were obtained from Harlan Sprague-Dawley. They were housed ( 3 hamsters/cage) under controlled temperature $\left(22^{\circ} \mathrm{C}\right)$ and lighting $(12: 12 \mathrm{~h}$ light/dark cycle). Animals were fed a standard rodent diet and permitted free access to autoclaved water. For adenoviral infection experiments, hamsters were fully anesthetized with isoflurane mixed with pure oxygen and injected with $2 \times 10^{10}$ pfu of Ad-hamACSL3 $(n=6)$ or Ad-GFP adenovirus $(n=6)$ via retro-orbital sinus in a total volume of $200 \mu \mathrm{l}$ of sterile PBS. Fasting serum was collected at days $0,4,7,11$ and 14 post-infection and liver tissue was obtained after sacrificing the hamsters at day 14 post-infection using procedures previously described $(8,19)$. All animal procedures and protocols were reviewed and approved by the Institutional Animal Care and Use Committee of the VA Palo Alto Health Care System.

Lipid measurement. Cellular, serum or hepatic TG, free fatty acid (FFA) and total phospholipids (PL) were determined using kits purchased from Stanbio Lab (Boerne, TX) and Wako Chemical GmbH (Neuss, Germany).

RNA isolation and real-time RT-PCR. Total RNA isolation from flash-frozen hamster liver and real-time RT-PCR were performed as previously described (8). Primer sequences are 
hamster ACSL3: forward: GGGCACCATTAGTTTGCTGT, reverse: CCGCTGTCCATTTTCATCTT; hamster $\beta$-actin: forward: TGACCGAGCGTGGCTACAG, reverse: CTTCT CTTTGATGTCACGCACAAT.

Western blot analysis. Western blot analysis was performed as previously described (8). Rabbit polyclonal anti-ACSL3 antibody was developed commercially against the C-terminal end of the hamster ACSL3 protein (THYQADIERMYGRK) and its characterization has been previously described (8). Anti-Flag M2 and anti- $\beta$-actin AC-15 antibodies were purchased from Sigma.

Immunofluorescent microscopy. HepG2 cells were seeded at $1 \times 10^{4}$ cells per well in 8-well chamber slides (Lab-Tek ${ }^{\circledR}$, IL). For adenoviral transduction, next day cells were transduced with the Ad-ham-ACSL3-Flag virus at a MOI of 50 and allowed to grow for $48 \mathrm{~h}$ prior to immunofluorescent staining. To detect endogenous or exogenously overexpressed hamACSL3 protein, cells were first fixed with $4 \%$ paraformaldehyde in PBS for $15 \mathrm{~min}$ at room temperature. After washing in ice-cold PBS twice, we added $0.2 \%$ Triton X-100 in PBS to permeabilize the cells for $10 \mathrm{~min}$ at room temperature, followed by washing with PBS three times for another $5 \mathrm{~min}$. Cells were then blocked with $10 \%$ goat serum in PBS for $30 \mathrm{~min}$ at room temperature. Next, cells were incubated with a mixture of two primary antibodies in $1 \%$ BSA in PBS for $1 \mathrm{~h}$ at room temperature. Cells were washed in PBS for 3 times and incubated in 1\% BSA in PBS containing two fluorescencelabeled secondary antibodies for $1 \mathrm{~h}$ at room temperature. Finally, cells were washed three times for $5 \mathrm{~min}$ and the detached glass slides were mounted with coverslip using a drop of mounting media (Biomedia, CA). Confocal microscopy was performed using a Zeiss LSM 510 Confocal Laser Scanning Microscope. Primary antibodies used were anti-GRP78 mouse monoclonal antibody (BD Transduction Laboratory, CA), anti-VDAC mouse monoclonal antibody (CalBiochem, CA), anti-Flag M2 mouse monoclonal antibody (Sigma, MO), anti-ACSL3 rabbit polyclonal antibody (8), anti-GRP78 rabbit polyclonal antibody (Abcam, MA), anti-VDAC rabbit polyclonal antibody (Cell Signaling Technology, MA). Secondary antibodies used were anti-mouse Alexa Fluor 488, anti-mouse Alexa Fluor 633 and anti-rabbit Alexa Fluor 546 (Invitrogen, CA).

Statistical analysis. Data were analyzed by Student's two-tailed $\mathrm{t}$-test. A $\mathrm{p}<0.05$ is considered statistically significant.

\section{Results}

ACSL enzymatic activity increases after hamster ACSL3 overexpression in HepG2 cells. HepG2 cells were transduced with either a control Ad-GFP or Ad-hamACSL3 virus expressing a C-terminal Flag-tagged hamster ACSL3 for $48 \mathrm{~h}$ at different doses. ACSL enzymatic activity was subsequently measured using $\left[{ }^{3} \mathrm{H}\right]$-palmitic acid. When compared with Ad-GFP control, Ad-hamACSL3 transduction increased ACSL activity 60,144 and $115 \%$ at MOI of 10, 50 and 100, respectively (Fig. 1A). Western blot analyses using anti-Flag antibody (Fig. 1B) and anti-ACSL3 antibody (Fig. 1C) confirmed that the Flag-tagged hamster ACSL3 protein was indeed overexpressed

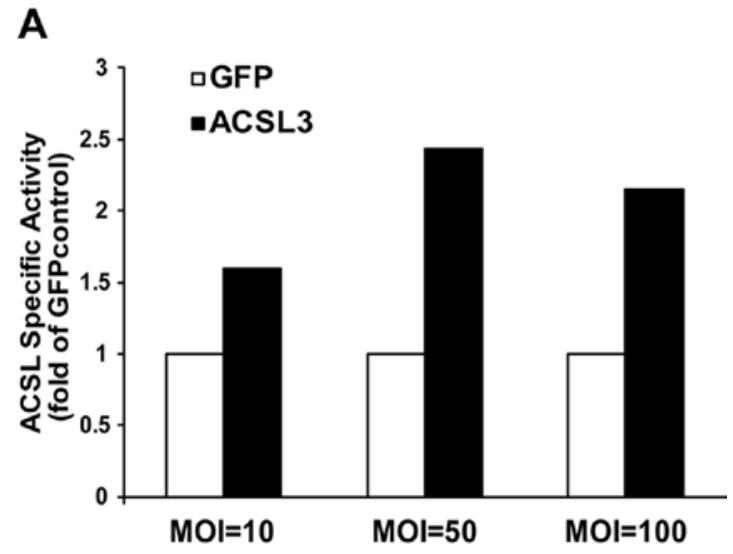

B

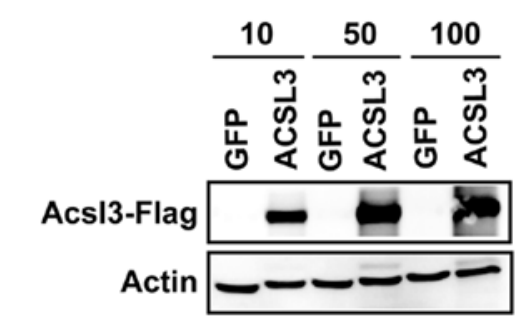

C

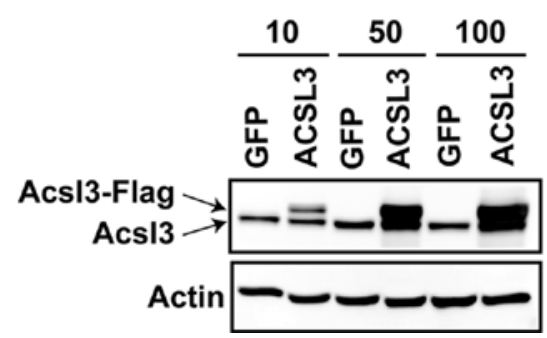

Figure 1. Acyl-CoA synthetase (ACSL) activity and ACSL3 overexpression in Ad-hamACSL3 and Ad-GFP adenovirus transduced HepG2 cells. (A) ACSL activity in HepG2 cells transduced with either control Ad-GFP or Ad-hamACSL3 adenovirus at MOI of 10, 50 and 100 for 48 h. (B) Western blot analysis of ectopically overexpressed hamACSL3 using anti-Flag M2 antibody in lysates prepared from HepG2 transduced with control Ad-GFP Ad-hamACSL3 at MOI of 10, 50 and 100 for $48 \mathrm{~h}$. $\beta$-actin was used as a loading control. (C) Western blot analysis of both endogenous human ACSL3 (lower band) and ectopically overexpressed hamACSL3 (upper band) using anti-ACSL3 antibody in above lysates. $\beta$-actin was used as a loading control.

in the transduced HepG2 cells and thereby responsible for the increase in total enzymatic activity.

Both endogenous and ectopically expressed ACSL3 localize to endoplasmic reticulum and mitochondria. It has been thought that the subcellular location of ACSL proteins could affect their functions in channeling FA into catabolic or anabolic pathways. It was not clear which subcellular compartment(s) ACSL3 is specifically localized to. By utilizing a highly specific anti-ACSL3 antibody and an anti-Flag antibody, we performed confocal microscopy to determine the intracellular localization of ACSL3 in HepG2 cells without and with adenovirus transduction. Anti-ACSL3 antibody can detect, as previously reported (8), both the endogenous human ACSL3 as well as the exogenously expressed hamster ACSL3 while anti-Flag antibody detects exclusively the overexpressed 

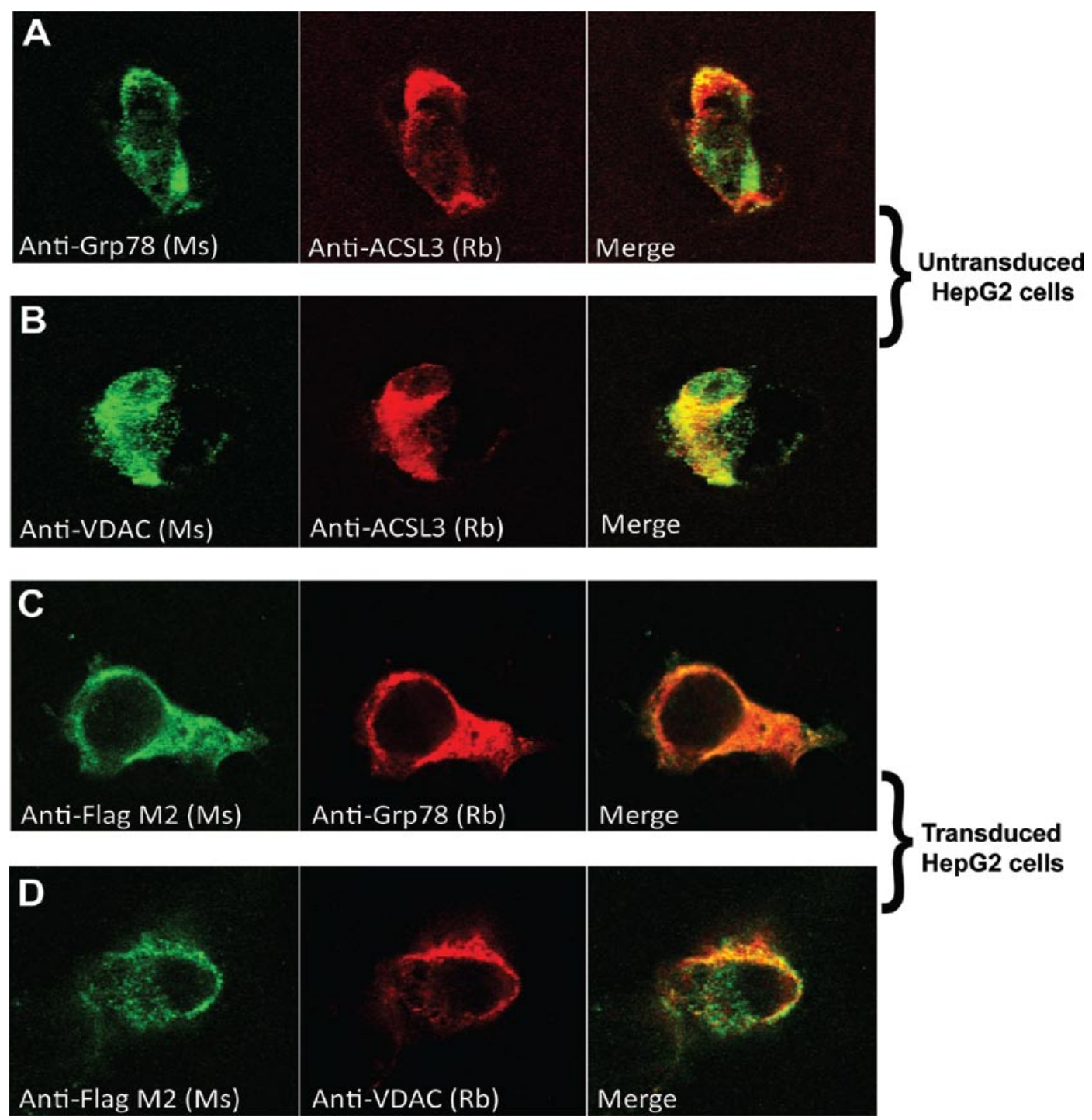

Figure 2. Both human and hamster ACSL3 proteins are localized at endoplasmic reticulum and mitochondria. (A) Untransduced HepG2 cells were subject to immunofluorescent staining using antibodies against Grp78 (endoplasmic reticulum marker) (mouse anti-Grp78 antibody) and ACSL3 (rabbit anti-ACSL3 antibody). (B) Untransduced HepG2 cells were subject to immunofluorescent staining using antibodies against VDAC (mitochondria marker) (mouse antiVDAC antibody) and ACSL3 (rabbit anti-ACSL3 antibody). (C) HepG2 cells transduced with Ad-hamACSL3 for $48 \mathrm{~h}$ were subject to immunofluorescent staining using antibodies against Grp78 (rabbit anti-Grp78 antibody) and Flag (mouse anti-Flag M2 antibody). (D) HepG2 cells transduced with Ad-hamACSL3 for $48 \mathrm{~h}$ were subject to immunofluorescent staining using antibodies against VDAC (rabbit anti-VDAC antibody) and Flag (mouse anti-Flag M2 antibody).

hamster ACSL3 (8). As shown in Fig. 2A and B, in untransduced HepG2 cells ACSL3 was colocalized with the endoplasmic reticulum (ER) marker Grp78 and also with the mitochondria marker VDAC. Analogous to anti-ACSL3 antibody, anti-Flag antibody showed the colocalization of exogenously expressed hamster ACSL3 with the ER marker Grp78 and the mitochondria marker VDAC in Ad-hamACSL3 transduced HepG2 cells (Fig. 2C and D). Thus, these data indicate that both human and hamster ACSL3 proteins are localized in ER and mitochondria.

Ad-hamACSL3 reduces cellular TG content and total phospholipids in HepG2 cells. To determine the effect of overexpression of hamster ACSL 3 on cellular lipid metabolism, we transduced HepG2 cells with Ad-hamACSL3 or control Ad-GFP virus at an MOI of 10 . After $72 \mathrm{~h}$ of transduction, cellular lipids were extracted and assayed for TG and total phospholipids. Overexpression of ACSL3 lowered the TG content of HepG 2 cells by $21 \%$ ( $\mathrm{p}<0.01$ ) (Fig. 3A). In addition, total phospholipids of Ad-hamACSL3 infected HepG2 cells was $17 \%$ lower $(\mathrm{p}<0.01)$ than that of control virus transduced cells (Fig. 3B). The cellular proliferation and morphology of HepG2 cells were not affected by Ad-hamACSL3 transduction.

Overexpression of ACSL3 reduces serum TG and FFA in hamsters. To further validate the TG-lowering effect of ACSL3 in vivo, we injected the Ad-hamACSL3 or control Ad-GFP virus into hamsters fed a standard rodent diet. To this end, overexpression of hamster ACSL3 mRNA and protein in liver was confirmed by real-time RT PCR and Western blotting at day 14 post-Ad-hamACSL3 infection. As shown in Fig. 4A, sustained increase in ACSL3 mRNA, when compared to control, 
A

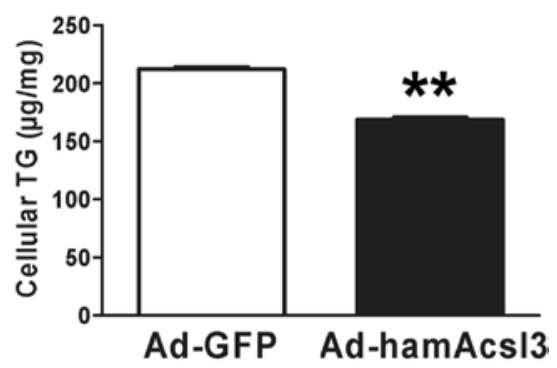

B

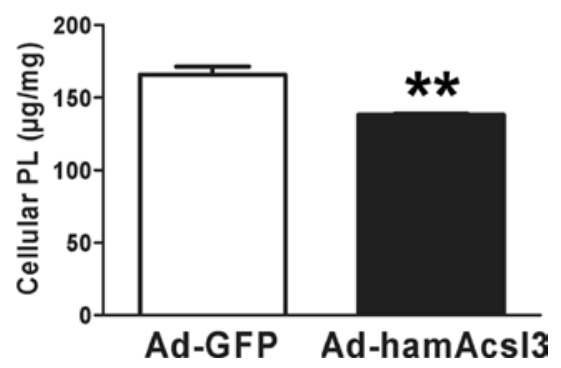

Figure 3. Effects of hamACSL3 overexpression on cellular lipid levels in HepG2 cells. (A) Cellular TG level in HepG2 cells transduced with control Ad-GFP or Ad-hamACSL3 at MOI of 10 for $72 \mathrm{~h}$. (B) Cellular PL level in HepG2 cells transduced with control Ad-GFP or Ad-hamACSL3 at MOI of 10 for $72 \mathrm{~h}$. Data are the mean $\pm \operatorname{SEM}(\mathrm{n}=3) .{ }^{* *} \mathrm{p}<0.01$. was observed at 2 weeks post-infection for $>2.0$-fold $(\mathrm{p}<0.05)$. Consistent with mRNA, protein expression of ACSL3-Flag appeared to last after 2 weeks of infection (Fig. 4B). Moreover, total hepatic ACSL3 protein levels determined by the antiACSL3 antibody increased 1.9 -fold $(\mathrm{p}<0.05)$ than GFP control (Fig. 4C and D). Ad-hamACSL3 infection, in turn, raised total hepatic ACSL activity by $14 \%$ when compared with Ad-GFP infected livers (Fig. 4E). In addition, we recorded post-infection body weight and food intake, but found no significant difference between ACSL3 and GFP control infected hamsters (data not shown). In agreement with in vitro findings, Ad-hamACSL3 viral infected hamsters exhibited lower serum TG level than that of Ad-GFP infected animals. Serum TG decreased as early as day 4 post-infection $(12 \%$ reduction) and reached lower levels at day 7 (16\% reduction, $\mathrm{p}<0.05)$ and day 11 (21\% reduction). It was then back to near control level (10\% reduction) at day 14 (Fig. 5A). The postinfection serum FFA levels reduced $29 \%(\mathrm{p}<0.05)$ at day 11 and $36 \%(\mathrm{p}<0.05)$ at day 14 in ACSL3 overexpressed hamsters (Fig. 5B). Unlike TG and FFA, serum PL levels did not appear to be significantly altered by Ad-hamACSL3 overexpression (Fig. 5C). Mirroring the changes in serum lipids, hepatic TG levels of Ad-hamACSL3 infected hamsters were $43 \%$ lower than that of the control animals $(\mathrm{p}<0.05)$ (Fig. 5D). There was a tendency toward decreased hepatic FFA in Ad-hamACSL3 infected hamsters, but it did not reach statistical significance due to individual sample variations (Fig. 5D). Additionally, a
A

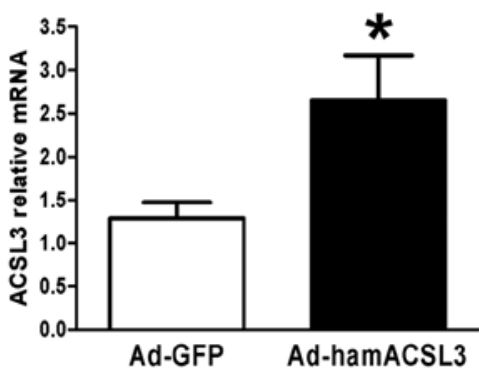

B

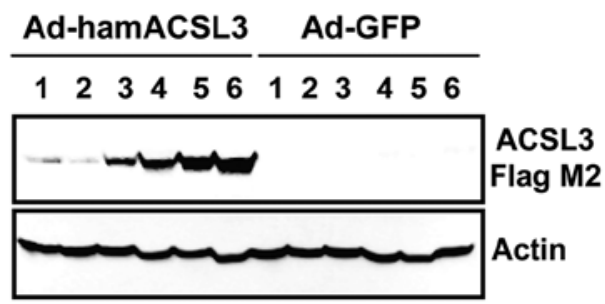

C

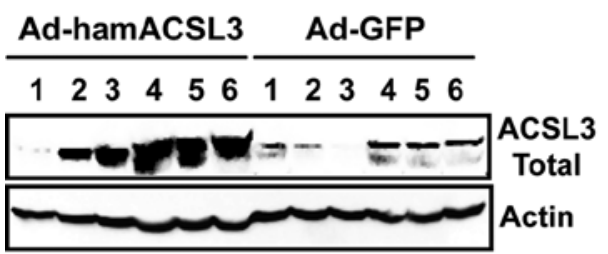

D

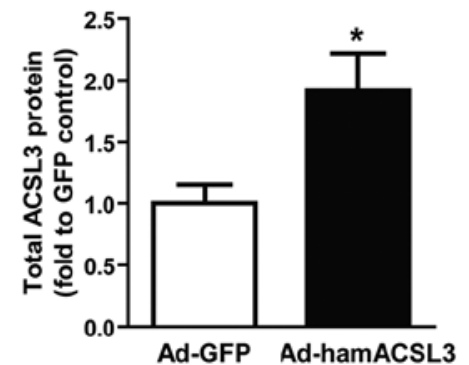

$\mathbf{E}$

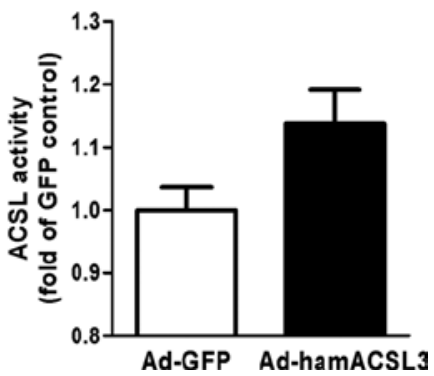

Figure 4. Hepatic ACSL3 expression and ACSL activity in hamsters infected with Ad-GFP or Ad-hamACSL3. Hamsters were infected with $2 \times 10^{10}$ pfu of Ad-hamACSL3 or Ad-GFP adenovirus via retro-orbital sinus as described in Materials and methods. Livers were harvested at day 14 post-infection. (A) Total ACSL3 mRNA levels were determined by real-time RT-PCR and normalized to $\beta$-actin. Data are the mean \pm SEM (n=6). *p<0.05. (B) Adenovirus-carried ACSL3-Flag protein expression was measured by Western blot analysis using anti-Flag M2 antibody. $\beta$-actin was used as a loading control. (C) Total liver ACSL3 protein expression was determined by Western blot analysis using anti-ACSL3 antibody. $\beta$-actin was used as a loading control. (D) Quantification of total liver ACSL3 protein expression. Data are the mean \pm SEM $(n=6)$. ${ }^{*} \mathrm{p}<0.05$. (E) Hepatic total ACSL enzymatic activity in hamsters infected with Ad-hamACSL3 or Ad-GFP adenovirus. Data are the mean \pm SEM $(n=3)$. 
A

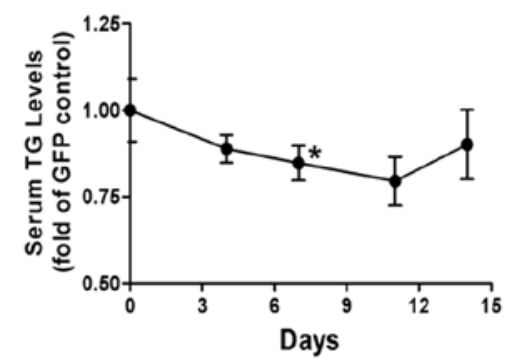

C

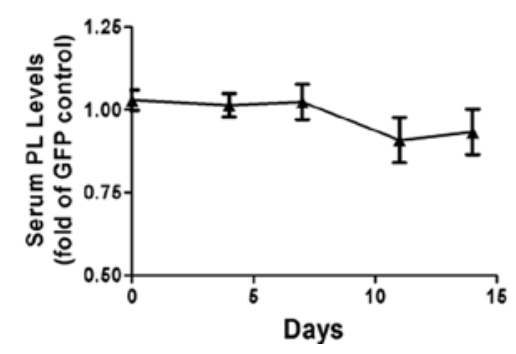

B

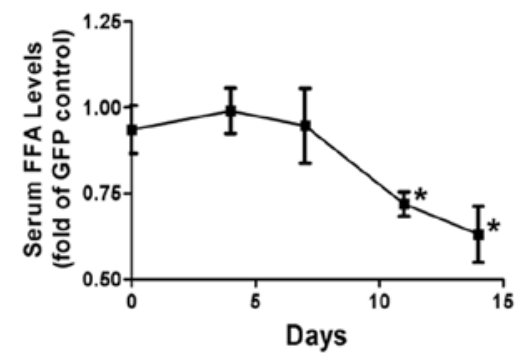

D

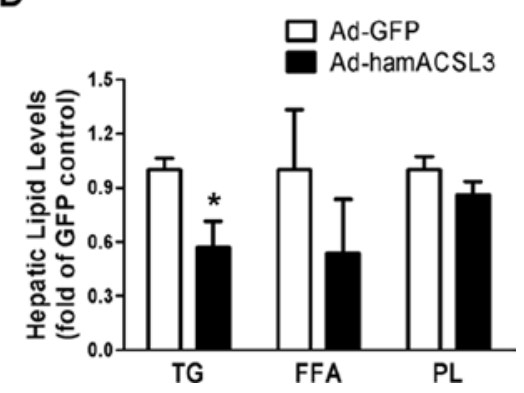

Figure 5. Effect of hamACSL3 overexpression on serum and liver lipids levels of hamsters infected with Ad-GFP or Ad-hamACSL3. (A) TG levels in hamster serum collected at $0,4,7,11$ and 14 days after infection with Ad-GFP or Ad-hamACSL3 adenovirus. Data (mean \pm SEM, n=5) are expressed as fold of GFP control at each time-point. *p<0.05. (B) FFA levels in hamster serum collected at 0, 4, 7, 11 and 14 days after infection with Ad-GFP or Ad-hamACSL3 adenovirus. Data (mean \pm SEM, $n=5$ ) are expressed as fold of GFP control at each time-point. * $<<0.05$. (C) PL levels in hamster serum collected at $0,4,7,11$ and 14 days after infection with Ad-GFP or Ad-hamACSL3 adenovirus. Data (mean \pm SEM, n=5) are expressed as fold of GFP control at each time-point. ${ }^{*} \mathrm{p}<0.05$. (D) TG, FFA and PL levels in hamster liver harvested at day 14 post infection of Ad-GFP or Ad-hamACSL3 adenovirus. Data (mean \pm SEM, $\mathrm{n}=5$ ) are expressed as fold of GFP control. * $\mathrm{p}<0.05$.

slightly reduced level of liver PL (14\% reduction) were also observed (Fig. 5D).

Examination of expression of genes involved in hepatic lipid metabolism. To determine whether overexpression of ACSL3 could affect gene expression in liver, we examined, using real-time RT PCR, several groups of genes whose protein products are involved in regulation of liver lipid metabolism. These included fatty acid synthase (FAS), stearoyl-CoA desaturase-1 (SCD-1), diacylglycerol acyltransferase 2 (DGAT2), peroxisome proliferator-activated receptor $\gamma$ (PPAR $\gamma$ ), peroxisome proliferator-activated receptor $\alpha$ (PPAR $\alpha$ ), carnitine palmitoyltransferase 1a (CPT1a) and very long-chain acyl-CoA dehydrogenase (ACADVL). However, we observed no significant differences in gene expression between Ad-hamACSL3 and Ad-GFP infected hamster livers.

\section{Discussion}

Long-chain acyl-CoA synthetases (ACSLs), consisting of five isoforms in mammals, activate long-chain fatty acids to form acyl-CoAs, which serve as substrates for both anabolic and catabolic pathways. In recent years, the functional involvement of some ACSLs, like ACSL1 $(14,15,17)$ and ACSL5 (13), as regulators for fatty acid partitioning has emerged. However, there is still lack of studies directly addressing the role of the other ACSL isoforms in lipid metabolism in vivo.

The present study was designed to investigate the role of ACSL3 in regulating hepatic and whole body lipid metabolism in vivo through adenoviral-mediated liver-targeted overexpression. Our in vitro data showed that overexpression of hamster ACSL3 in adenoviral transduced HepG2 cells led to a dose-dependent increase in total ACSL activity, indicating that the exogenously expressed hamster ACSL3 protein is indeed fully functional in a context of human liver-derived cells. Further, consistent with our previous study on human ACSL3 (19), overexpression of its hamster counterpart was of physiological importance and had functional consequence since it led to a decrease in cellular TG as well as PL content in human HepG2 cells.

Previous studies using subcellular fractionation techniques found ACSL3 protein in a lipid droplet-enriched fraction isolated from human Huh7 hepatoma cells $(25,26)$. Additionally, proteomic studies revealed that ACSL3 is present in lipid body in 3T3 adipocytes (27) and A431 human squamous epithelial carcinoma cells (28). In order to directly examine the intracellular localization of ACSL3, we developed a robust rabbit polyclonal antibody that can recognize the ACSL3 protein across different species, including human, rat, mouse and hamster, in a highly specific manner (8). By use of this antibody and the confocal technology, we provided the first demonstration of subcellular localization of the endogenous human ACSL3 protein and the exogenously expressed hamster ACSL3 on endoplasmic reticulum and mitochondria. It is worth mentioning that ACSL1 was also identified on various subcellular locations including endoplasmic reticulum, microsome, mitochondria, cytosol and GLUT4 vesicles (11,29-31), and possessed somehow opposite functions on FA channeling in different tissues, favoring TG synthesis in liver $(14,15)$ while promoting mitochondria $\beta$-oxidation in adipocytes (18). Therefore, it is reasonable to speculate that, the mitochondria-localized ACSL3 could be responsible for 
its lipid lowering effects through channeling FAs toward mitochondria $\beta$-oxidation in HepG 2 cells and in liver of hamsters.

The most significant finding of this study was the reductive effects of ACSL3 on hepatic TG and serum TG and FFA levels in vivo. Taking advantage of the liver-homing characteristics of adenovirus, we were able to achieve high levels of liverspecific overexpression of ACSL3 in hamster after 2 weeks post-infection. It is currently unfeasible to separately measure ACSL3 activity from all other ACSLs. Therefore, a 14\% increase of total liver ACSL activity post-infection indeed was an indirect reflection of a functional overexpression of virus-carried ACSL3 in hamster liver. Above all, ACSL1 but not ACSL3 is the dominant form in liver. Additionally, overexpression of ACSL3 had no significant effect on hamster body weight or food intake, suggesting that the reduction of TG and FFA in serum and live after virus infection was mainly contributed by ACSL3 overexpression. Despite the changes in lipid levels of plasma and liver, we did not observe significant changes in hepatic mRNA levels of several genes whose products are involved in TG synthesis or fatty acid $\beta$-oxidation, suggesting that the observed effects of Ad-hamACSL3 on lipid metabolism were independent of changes in lipogenic or oxidative gene expression in hamster liver. The precise mechanism by which ACSL3 overexpression leads to TG and FFA reduction in serum and liver remains unclear. We have previously reported that overexpression of human ACSL3 protein in HepG2 cells caused a $32.5 \%$ increase of fatty acid oxidation along with a $67 \%$ reduction of cellular TG content (19). Therefore, it is highly likely that liver-targeted overexpression of ACSL 3 could facilitate fatty acid $\beta$-oxidation in hamster liver cells in vivo, which, in turn, leads to serum and hepatic TG and FFA reduction. While some evidence reported by others $(26,32)$ tend to favor a lipogenic role of ACSL3 in lipid metabolism, the data from this current in vivo study and our previous in vitro studies suggest a catabolic role of ACSL3 in hepatic lipid metabolism.

In conclusion, by utilizing adenoviral-mediated gene delivery approach we were able to increase ACSL3 expression specifically in liver tissue. This allowed us to obtain the first in vivo evidence demonstrating a TG-reducing function of isozyme ACSL3 of the ACSL family in liver of hamsters.

\section{Acknowledgements}

The Department of Veterans Affairs (Office of Research and Development, Medical Research Service) and the National Center for Complementary and Alternative Medicine (grants: 1RO1 AT002543-01A1 and 1R21AT003195-01A2) supported this work.

\section{References}

1. Soupene E and Kuypers FA: Mammalian long-chain acyl-CoA synthetases. Exp Biol Med 233: 507-521, 2008.

2. Fujino $\mathrm{T}$ and Yamamoto $\mathrm{T}$ : Cloning and functional expression of a novel long-chain acyl-CoA synthetase expressed in brain. $\mathrm{J}$ Biochem 111: 197-203, 1992.

3. Fujino T, Kang MJ, Suzuki H, Iijima $H$ and Yamamoto T: Molecular characterization and expression of rat acyl-CoA synthetase 3. J Biol Chem 271: 16748-16752, 1996.
4. Kang MJ, Fujino T, Sasano H, et al: A novel arachidonatepreferring acyl-CoA synthetase is present in steroidogenic cells of the rat adrenal, ovary, and testis. Proc Natl Acad Sci USA 94: 2880-2884, 1997.

5. Oikawa E, Iijima H, Suzuki T, et al: A novel acyl-CoA synthetase, ACS5, expressed in intestinal epithelial cells and proliferating preadipocytes. J Biochem 124: 679-685, 1998.

6. Suzuki H, Kawarabayasi Y, Kondo J, et al: Structure and regulation of rat long-chain acyl-CoA synthetase. J Biol Chem 265: 8681-8685, 1990 .

7. Mashek DG, Li LO and Coleman RA: Rat long-chain acyl-CoA synthetase mRNA, protein, and activity vary in tissue distribution and in response to diet. J Lipid Res 47: 2004-2010, 2006.

8. Wu M, Liu H, Chen W, Fujimoto Y and Liu J: Hepatic expression of long-chain acyl-CoA synthetase 3 is upregulated in hyperlipidemic hamsters. Lipids 44: 989-998, 2009.

9. Igal RA, Wang P and Coleman RA: Triacsin C blocks de novo synthesis of glycerolipids and cholesterol esters but not recycling of fatty acid into phospholipid: evidence for functionally separate pools of acyl-CoA. Biochem J 324: 529-534, 1997.

10. Kim JH, Lewin TM and Coleman RA: Expression and characterization of recombinant rat Acyl-CoA synthetases 1, 4, and 5 . Selective inhibition by triacsin $\mathrm{C}$ and thiazolidinediones. J Biol Chem 276: 24667-24673, 2001.

11. Lewin TM, Kim J-J, Granger DA, Vance JE and Coleman RA: Acyl-coA synthetase isoforms 1, 4 and 5 are present in different subcellular membranes in rat liver and can be inhibited independently. J Biol Chem 276: 24674-24679, 2001.

12. Van Horn CG, Caviglia JM, Li LO, Wang S, Granger DA and Coleman RA: Characterization of recombinant long-chain rat acyl-CoA synthetase isoforms 3 and 6: identification of a novel variant of isoform 6. Biochemistry 44: 1635-1642, 2005.

13. Mashek DG, McKenzie MA, van Horn CG and Coleman RA: Rat long chain acyl-CoA synthetase 5 increases fatty acid uptake and partitioning to cellular triacylglycerol in McArdle-RH7777 cells. J Biol Chem 281: 945-950, 2006.

14. Parkers HA, Preston E, Wilks D, et al: Overexpression of acyl-CoA synthetase-1 increases lipid deposition in hepatic (HepG2) cells and rodent liver in vivo. Am J Physiol Endocrinol Metab 291: E737-E744, 2006.

15. Li LO, Mashek DG, An J, Doughman SD, Newgard CB and Coleman RA: Overexpression of rat long chain acyl-CoA synthetase 1 alters fatty acid metabolism in rat primary hepatocytes. J Biol Chem 281: 37246-37255, 2006.

16. Chiu HC, Kovacs A, Ford DA, et al: A novel mouse model of lipotoxic cardiomyopathy. J Clin Invest 107: 813-822, 2001.

17. Li LO, Ellis JM, Paich HA, et al: Liver-specific loss of long chain acyl-CoA synthetase-1 decreases triacylglycerol synthesis and beta-oxidation and alters phospholipid fatty acid composition. J Biol Chem 284: 27816-27826, 2009.

18. Ellis JM, Li LO, Wu PC, et al: Adipose acyl-CoA synthetase-1 directs fatty acids toward beta-oxidation and is required for cold thermogenesis. Cell Metab 12: 53-64, 2010.

19. Zhou Y, Abidi P, Kim A, Chen W, Huang TT, Kraemer FB and Liu J: Transcriptional activation of hepatic ACSL3 and ACSL5 by oncostatin M reduces hypertriglyceridemia through enhanced beta-oxidation. Arterioscler Thromb Vasc Biol 27: 2198-2205, 2007.

20. Minekura H, Fujino T, Kang MJ, Fujita T, Endo Y and Yamamoto TT: Human acyl-coenzyme A synthetase 3 cDNA and localization of its gene (ACS3) to chromosome band 2q34-q35. Genomics 42: 180-181, 1997.

21. Cao A, Li H, Zhou Y, Wu M and Liu J: Long chain acyl-CoA synthetase-3 is a molecular target for peroxisome proliferatoractivated receptor delta in HepG2 hepatoma cells. J Biol Chem 285: 16664-16674, 2010.

22. Aurisicchio L, Delmastro P, Salucci V, et al: Liver-specific alpha 2 interferon gene expression results in protection from induced hepatitis. J Virol 74: 4816-4823, 2000.

23. Kozarsky KF, McKinley DR, Austin LL, Raper SE, StratfordPerricaudet LD and Wilson JM: In vivo correction of low density lipoprotein receptor deficiency in the Watanabe heritable hyperlipidemic rabbit with recombinant adenoviruses. J Biol Chem 269: 13695-13702, 1994.

24. Spady DK, Cuthbert JA, Willard MN and Meidell RS: Adenovirus-mediated transfer of a gene encoding cholesterol 7 alpha-hydroxylase into hamsters increases hepatic enzyme activity and reduces plasma total and low density lipoprotein cholesterol. J Clin Invest 96: 700-709, 1995. 
25. Fujimoto Y, Itabe H, Sakai J, et al: Identification of major proteins in the lipid droplet-enriched fraction isolated from the human hepatocyte cell line HuH7. Biochim Biophys Acta 1644: 47-59, 2004.

26. Fujimoto Y, Itabe H, Kinoshita T, et al: Involvement of ACSL in local synthesis of neutral lipids in cytoplasmic lipid droplets in human hepatocyte HuH7. J Lipid Res 48: 1280-1292, 2007.

27. Brasaemle DL, Dolios G, Shapiro L and Wang R: Proteomic analysis of proteins associated with lipid droplets of basal and lipolytically stimulated 3T3-L1 adipocytes. J Biol Chem 279: 46835-46842, 2004

28. Umlauf E, Csaszar E, Moertelmaier M, Schuetz GJ, Parton RG and Prohaska R: Association of stomatin with lipid bodies. J Biol Chem 279: 23699-23709, 2004.
29. Gargiulo CE, Stuhlsatz-Krouper SM and Schaffer JE: Localization of adipocyte long-chain fatty acyl-CoA synthetase at the plasma membrane. J Lipid Res 40: 881-892, 1999.

30. Sleeman MW, Donegan NP, Heller-Harrison R, Lane WS and Czech MP: Association of acyl-CoA synthetase-1 with GLUT4containing vesicles. J Biol Chem 273: 3132-3135, 1998.

31. Wang Y, Guo W, Zang Y, et al: Acyl Coenzyme A synthetase regulation: putative role in long-chain acyl coenzyme A partitioning. Obes Res 12: 1781-1788, 2004.

32. Bu SY, Mashek MT and Mashek DG: Suppression of long chain acyl-CoA synthetase 3 decreases hepatic de novo fatty acid synthesis through decreased transcriptional activity. J Biol Chem 284: 30474-30483, 2009. 